International Journal of Dental Research, 8(2) (2021) 49-53
International Journal of Dental Research
SPC
Website: www.sciencepubco.com/index.php/IJDR
Research paper

\title{
Creating smiles the lip repositioning way a case report
}

\author{
Dr. Nikhila Chandramohan ${ }^{1 *}$, Dr Lalith Vivekanand ${ }^{2}$, Dr. Swetha $\mathrm{A}^{2}$, Dr. Faiza Fathima ${ }^{2}$ \\ ${ }^{1}$ Postgraduate, Department of Periodontics, M R Ambedkar Dental College, $1 \backslash 36$, \\ Cline Road, Balaji Layout, Cooke town, Bangalore, Karnataka-560005, INDIA \\ ${ }^{2}$ Reader, M R Ambedkar dental college, Bangalore, Karnataka \\ *Corresponding author E-mail: niki_cm91@hotmail.com
}

\begin{abstract}
A beautiful smile comprises a perfect balance of the white and pink. A gummy smile has been a prevalent esthetic disorder with varied etiologies including of skeletal, dento-alveolar, or soft-tissue origin. It can be managed by a variety of treatment modalities after appropriate diagnosis and treatment planning. If it is due to jaw deformities, altered passive eruption or tooth malpositioning, the corrective measures incorporate orthognathic surgery and orthodontic treatment. However, this requires hospitalization and entails significant discomfort. Lip repositioning is a simple and predictable technique that restricts the muscle pull of the elevator lip muscles thereby reducing the gingival display. This case report demonstrates the successful management of a young patient with moderate gummy smile using the conventional LR surgery thereby proving to be a promising alternative that is less invasive in nature and with minimal postop complications. At 6 months, the results were stable.
\end{abstract}

Keywords: Esthetic; Gingival Display; Gummy Smile; Lip Repositioning.

\section{Introduction}

Smile, a person's ability to express a range of emotions with the structure and movement of the teeth and lips, can often determine how well a person can function in a society. Esthetics or the concept of beauty is determined by cultural, social, economic and personal factors. An aesthetic smile though subjective, clinically requires the optimal inter-relationship between the teeth, surrounding oral structures and periodontal complex. When a disharmony exists between these three components, the result is a smile that is perceived as unaesthetic.

In the present era, wherein social media has become the principle component of our daily lives, we see a growing esthetic demand among patients who are well informed and aware about the available options for improving their smile. Various factors that need to be considered in smile designing include tooth alignment, contact areas, surface texture, emergence profile, restorative marginal form, embrasures in relation to the gingival margins and interdental papillae, gingival display, etc. It is commonly observed that patients, who exhibit an upper lip that lies far enough above the maxillary gingival zenith, perceive it as unattractive. This condition is known as excessive gingival display (EGD). According to Peck et al. (1992), EGD otherwise called the "gummy smile," (GS) is defined as more than 2 mm of gingival exposure when a subject is smiling. ${ }^{1}$ An excessive gingiva-to-lip distance of $4 \mathrm{~mm}$ or more is classified as "unattractive" by lay people and general dentists.[2].

If it is due to the sole etiology of altered eruption - passive or active, short clinical crown heights may be managed with anterior gingivectomy or crown lengthening only. It is a well documented treatment modality that mainly involves soft tissue and hard tissue resection and is proved to be effective in restoring normal tooth dimensions and dentogingival relationships.[3] Other possible etiologies include i) vertical maxillary excess (VME) with incompetent or hypermobile upper lip (HUL), wherein skeletal Class II relationships and vertical growth patterns are presented, ii) compensatory eruption of maxillary teeth with concomitant coronal migration of attachment apparatus including the gingival margins; in such cases surgical intervention such as Le Fort osteotomy, orthodontic intrusion, and/or osseous crown lengthening may have to be performed.[4] Besides requiring significant time and financial investments, these therapies have multiple inherent risks, chances of postoperative complications and the results may vary.[5] The average length of the upper lip is about 20-22 mm in young females and 22-24 mm in young males, and GS has been associated with a short upper lip.[6] HUL also results in GS that is due to the hyperactivity of the elevator muscles of the lip. The diagnosis of HUL is usually made when there is normal upper-lip length and equal facial thirds. Some case reports have suggested that injection of botulinum toxin can be effective in the treatment of hypermobility of the upper lips.[7] Hence during patient examination establishing the etiology responsible for EDS is indispensable to achieve the desired outcome.

The need of the hour was an effort to correct GS by the manipulation and repositioning of the lip and soft tissues inferiorly. A surgical technique for doing exactly this has been developed and is known as lip repositioning surgery (LRS). It can limit the retraction of the elevator muscles of the lip which is achieved by removing a strip of mucosa from the maxillary buccal vestibule and creating a partialthickness flap between the mucogingival junction and the upper lip musculature. The lip mucosa is then sutured to the mucogingival line, resulting in a shallow vestibule, restricted muscle pull, thereby obscuring the excessive gingival display during smiling. Moderate gingival 
display that is not skeletal in origin and vertical maxillary excess that ranges between 4 and $8 \mathrm{~mm}$ can be effectively treated by surgical repositioning of upper lip limiting the retraction muscles such as zygomaticus minor, orbicularis oris, levator anguli oris and levator labi oris. [8]

This technique was originally described as a cosmetic surgery by Rubinstein and Kostianovsky in 1973 for the correction of gummy smile caused by hypermobile lip. [9] A myriad of modifications was put forth in the following years by various clinicians for better results. It was reported to be a less invasive procedure comparatively with limited patient morbidity. The case presented here discusses the novel conventional lip repositioning procedure which was used for the correction of excessive gingival display, with 6 months follow-up.

\section{Case report}

A 25-year-old female patient reported to the Department of Periodontology, with a chief complaint of gummy smile. She wanted to minimize the gingival display in her smile. She presented a non-contributory medical history and there were no contraindications to surgical procedure.

On clinical examination, a moderate maxillary gingival display was observed (Fig I). Extraorally, bilateral symmetry of face and short upper lip was noted. With an exaggerated smile, intraorally the patient's teeth were visible from right maxillary second premolar to left maxillary second premolar with $6 \mathrm{~mm}$ of excessive gingival display (Fig II). Periodontal examination revealed a thick gingival biotype with an average probing depth of $3 \mathrm{~mm}$. The gingival line in the maxillary anterior sextant was found to be symmetrical and she had adequate width of attached gingiva. The maxillary anterior teeth had acceptable anatomic proportions. The surgical procedure was explained and informed consent was obtained from the patient.

\subsection{Surgical procedure}

Local Anesthetic (Lignocaine 2\% with 1:100,000 epinephrine) was administered in the vestibular mucosa and lip from maxillary right to left first molar. A sterile marker was used to outline the incisions on the dried tissues (Fig III). A partial thickness incision was made at the mucogingival junction from the mesial line angle of right first molar to mesial line angle left first molar. A second partial thickness incision, parallel to the first, was made in the labial mucosa $10 \mathrm{~mm}$ apical to the first. The incisions were connected at the first molar regions each, and resulted in an elliptical outline. The epithelium within the outlines of the incisions was removed, leaving the underlying connective tissue exposed (Fig IV). The tissue tags were removed. Ziv simon believed that the amount of tissue excised should be double the amount of gingival display that needs to be reduced with a maximum of 10-12 mm of tissue excised. [4] Care was taken to avoid damage to any minor salivary glands in the submucousal region. Bleeding was well controlled. The parallel incision lines were approximated with interrupted stabilization sutures, first at the midline followed by the flap ends next to the maxillary molars. Thereafter other remaining locations along the borders of incision were approximated with continuous interlocking sutures (Fig V). This was to ensure the proper alignment of lip midline with the midline of the teeth (Fig VI). Patient was prescribed antibiotics and analgesics for five days and ice pack application externally. The patient was instructed to rinse gently with $0.2 \%$ chlorhexidine gluconate twice daily for 2 weeks, to avoid any vigorous lip movements or pulling of the lips to see the surgical site and to minimize smiling and talking for a week. Postoperative healing was uneventful and no complications were reported. Patient reported a tense feeling on the upper lip and slight tenderness while smiling and speaking for 2 weeks after the surgery. Sutures were removed at 2 weeks. The suture line healed in the form of a scar that was not apparent when the patient smiled as it was concealed under the upper lip mucosa (Fig VII). At 2 weeks follow-up, satisfactory reduction in patient's gingival display was achieved and these results were found to be stable at 6 months (Fig VIII).

\subsection{Patient satisfaction analysis}

This was done based on visual analog scale (VAS) rating at baseline ( $24 \mathrm{~h}$ after treatment) and at 2 weeks. Patient comfort score (PCS) was taken on a scale of $0-10,0$ being no discomfort and 10 being unbearable discomfort. Patient esthetic score (PES) was recorded with 0 being poor esthetics and 10 being excellent esthetics. The PCS was recorded as 2 and PES as 9.

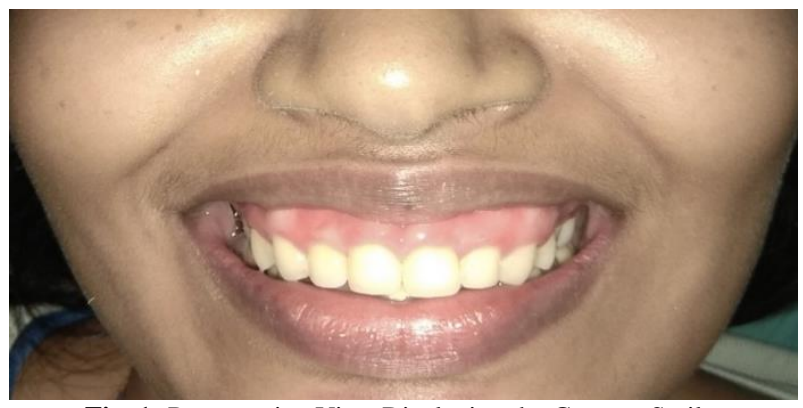

Fig. 1: Preoperative View Displaying the Gummy Smile.

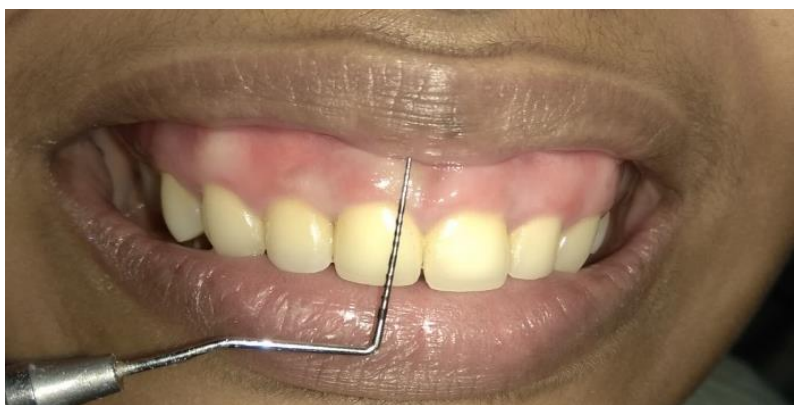

Fig. 2: 6mm Gingival Display as Measured Using UNC-15 Probe. 


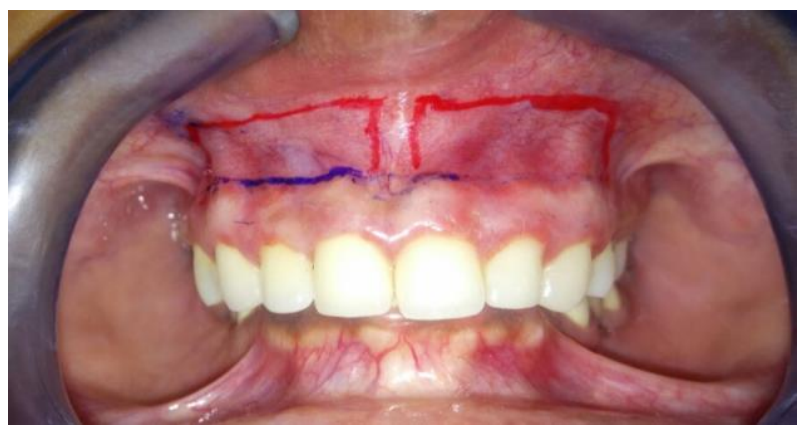

Fig. 3: 2 Parallel Incision Lines Marked with Sterile Marker.

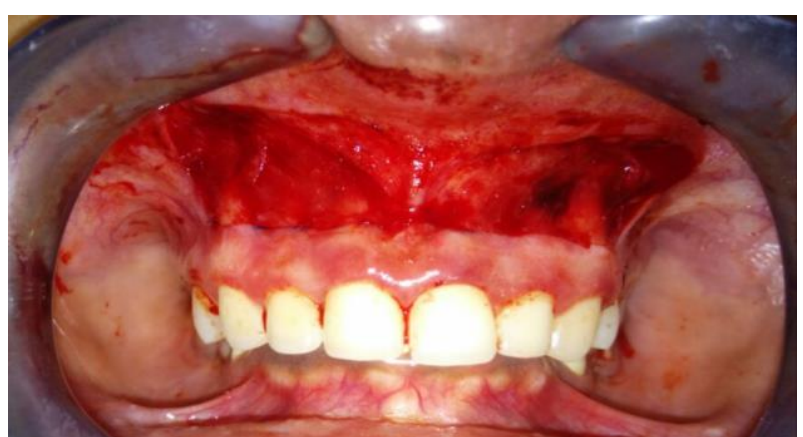

Fig. 4: Partial Thickness Flap Raised and Strip of Epithelium Removed Adjoining the Two Incision Lines to Expose the Underlying Connective Tissue.

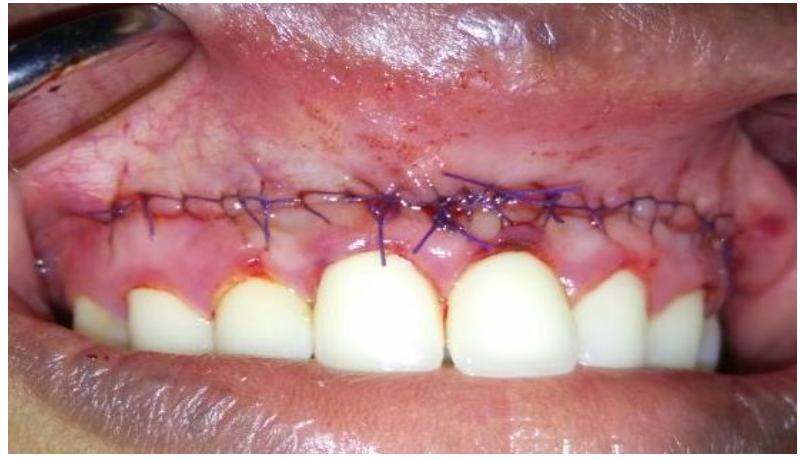

Fig. 5: Suturing Done with Continuos Interlocking Sutures.

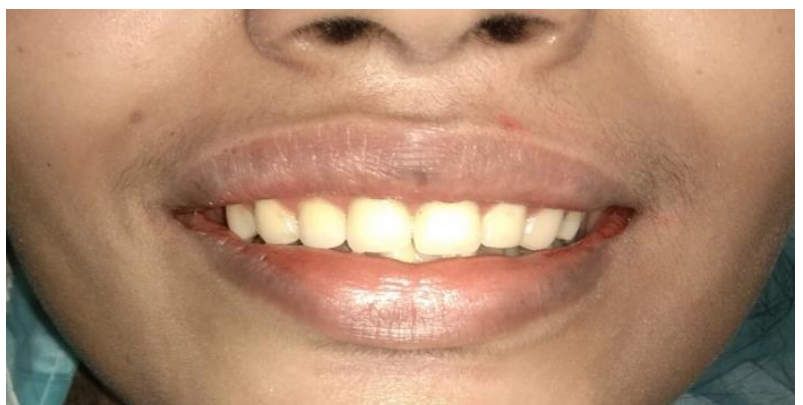

Fig. 6: Immediate Post Op View While Smiling

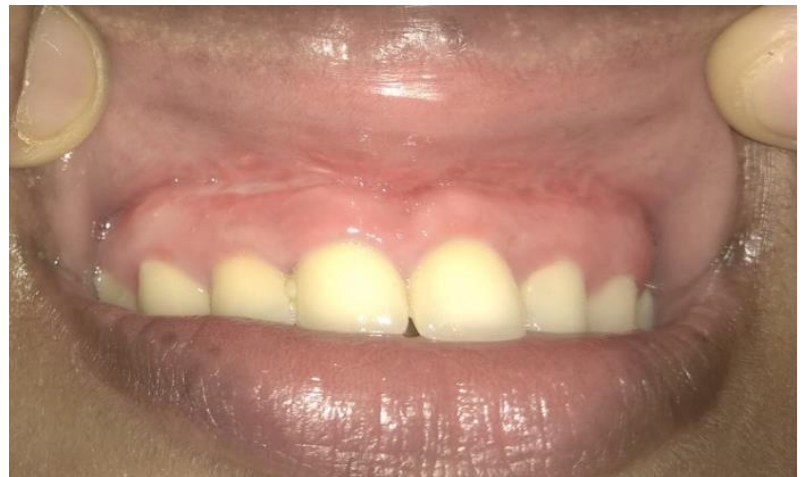

Fig. 7: Healed Suture Line. 


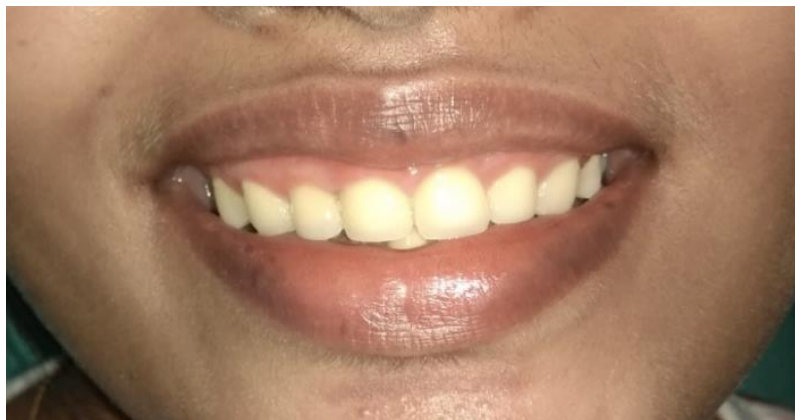

Fig. 7: Esthetically Enhanced Smile at 6 Month Follow Up.

\section{Discussion}

This report documents the use of LRS for the management of GS. About 2-3 mm of the exposed gingiva while smiling is cosmetically acceptable; however, more than this is considered as "Gummy smile". [10] GS has been associated with various conditions, and the literature is replete with numerous treatment modalities for the correction of it. Invasive procedures such as myotomy, myectomy, and orthognathic surgery have been indicated for the correction of GS, whereas there are also conservative techniques such as lip repositioning, crown lengthening, or the use of botulinum toxin, which have promising results.

Lip repositioning has gained a lot of impetus over the recent years after its inception in 1973 by Rubinstein and Kostianovsky. [9] This has become a chosen treatment modality because of the shorter, less aggressive approach, and it is shown to have lesser postoperative complications when compared to orthognathic surgery. A recent systematic review by Tawfik et al. in 2018 concluded that LPS successfully improved EGD by a mean of $3.4 \mathrm{~mm}$, suggesting it to be a successful approach for the treatment of GS, especially for patients with minor discrepancies desiring a less invasive alternative to orthognathic surgery and a more immediate and enduring result when compared to orthodontics and botox treatment. [11] Accurate diagnosis and a pertinent case selection is critical for the success of any LR procedure. Contraindications to LRS include the presence of a minimal zone of attached gingiva, which can create difficulties in flap design, stabilization, and suturing, and severe VME (>8 mm of gingival display). [4], [8].

In this case report, we present a case of a 25 year old female patient who is concerned regarding the esthetics of her smile. She desired lesser gingival display in her smile. Among the various options presented to her, patient agreed for LPS due to the less invasive nature. She had a moderate gingival display and an even gingival contour. The skeletal relationships were favorable. The conventional LPS was performed and a satisfactory reduction in the gingival display was achieved. At 6 months follow up, the results were stable.

There are numerous case reports in literature that have supported the application of LPS in GS correction and have shown to display esthetically pleasing smiles. One of the most frequently encountered demerit of this procedure is relapse, after a year or even earlier. Litton and Fournier (1979) re-advocated the technique given by Rubinstein and Kostianovsky, by including elevator muscle detachment in cases with a short upper lip. [12] This technique was further improvised by Miskinyar (1983), with the addition of myectomy and partial resection of either one or both of the levator labii superioris muscles bilaterally.[13] Ellenbogen and Swara (1984) reported success in limiting lip elevation on smiling (maximum correction, $6 \mathrm{~mm}$ ) by partially transecting the lip elevator muscles and implanting a silicone spacer. [14] Most recently, Ishida et al 2010 reported a significant reduction in gingival exposure (a mean $3.31 \mathrm{~mm}$ at 6 months ) in 14 patients treated with levator labii superioris myotomy, subperiosteal dissection, and frenectomy. [15] Advocating a nonsurgical approach, Polo (2008) reported successful temporary management of patients with a hyperfunctional upper lip using botulinum toxin type A. In perhaps his most telling observation, he states "improvement in self-esteem changes the scope of several of these cosmetic procedures to another level: therapeutic". [16], p.195 These reports have shown stable results in 6-month follow-up, but very few have shown results of long term. A systematic review also quoted that further studies are required to properly evaluate the surgical approach and the stability of this procedure. No postoperative complications, other than mild discomfort for 24 to 48 hours, were reported by the patient. Patient's satisfaction was quite evident from the PES and PCS. Miskinyar noted one patient with 2.5 months unilateral paresthesia [13], and Rosenblatt reported one patient with a mucocele that resolved without treatment [8]. Relapse over long term cannot be ruled out. Future clinical trials to compare the esthetic results obtained from various techniques that are aimed at correcting GS, and also studies with long-term follow-up needs to be conducted to assess the stability of these treatment modalities, so as to enable clinicians to arrive at definitive treatment plan.

\section{Conclusion}

This case report portrays the effective management of GS with a unique technique, the Lip Repostioning Surgery. Our results show good stability at 6-month follow-up. The pros include its less invasive nature, fewer post-operative complications and a faster recovery compared to orthognathic surgery. Hence it is patient and clinician friendly and promises to be a viable alternative. Long-term follow-ups are warranted.

\section{Acknowledgements}

None

\section{References}

[1] Peck, S., Peck, L., \& Kataja, M. (1992). Some vertical lineaments of lip position. American journal of orthodontics and dentofacial orthopedics: official publication of the American Association of Orthodontists, its constituent societies, and the American Board of Orthodontics, 101(6), 519524. https://doi.org/10.1016/0889-5406(92)70126-U.

[2] Kokich, V. O., Jr, Kiyak, H. A., \& Shapiro, P. A. (1999). Comparing the perception of dentists and lay people to altered dental esthetics. Journal of esthetic dentistry, 11(6), 311-324. https://doi.org/10.1111/j.1708-8240.1999.tb00414.x. 
[3] Lee E. A. (2004). Aesthetic crown lengthening: classification, biologic rationale, and treatment planning considerations. Practical procedures \& aesthetic dentistry: PPAD, 16(10), 769-780.

[4] Simon Z, Rosemblatt A, Dorfmann W (2007). Eliminating a gummy smile with surgicallip repositioning. The Journal of Cosmetic Dentistry, 23(1),100-108.

[5] Kim, S. G., \& Park, S. S. (2007). Incidence of complications and problems related to orthognathic surgery. Journal of oral and maxillofacial surgery: official journal of the American Association of Oral and Maxillofacial Surgeons, 65(12), 2438-2444. https://doi.org/10.1016/j.joms.2007.05.030.

[6] Peck, S., Peck, L., \& Kataja, M. (1992). The gingival smile lines. The Angle orthodontist, 62(2), 91-102. https://doi.org/10.1043/00033219(1992)062<0091:TGSL>2.0.CO;2

[7] Mostafa D. (2018). A successful management of sever gummy smile using gingivectomy and botulinum toxin injection: A case report. International journal of surgery case reports, 42, 169-174. https://doi.org/10.1016/j.ijscr.2017.11.055.

[8] Rosenblatt, A., \& Simon, Z. (2006). Lip repositioning for reduction of excessive gingival display: a clinical report. The International journal of periodontics \& restorative dentistry, 26(5), 433-437.

[9] Rubinstein A, Kostianovsky A. Cirugia estetica de la malformacion de la sonrisa. Pren Med Argent.1973;60:952.

[10] Silberberg, N., Goldstein, M., \& Smidt, A. (2009). Excessive gingival display--etiology, diagnosis, and treatment modalities. Quintessence international (Berlin, Germany: 1985), 40(10), 809-818.

[11] Tawfik, O. K., El-Nahass, H. E., Shipman, P., Looney, S. W., Cutler, C. W., \& Brunner, M. (2018). Lip repositioning for the treatment of excess gingival display: A systematic review. Journal of esthetic and restorative dentistry: official publication of the American Academy of Esthetic Dentistry ... [et al.], 30(2), 101-112. https://doi.org/10.1111/jerd.12352.

[12] Litton, C., \& Fournier, P. (1979). Simple surgical correction of the gummy smile. Plastic and reconstructive surgery, 63(3), $372-373$. https://doi.org/10.1097/00006534-197903000-00014.

[13] Miskinyar S. A. (1983). A new method for correcting a gummy smile. Plastic and reconstructive surgery, 72(3), 397-400. https://doi.org/10.1097/00006534-198309000-00027.

[14] Ellenbogen, R., \& Swara, N. (1984). The improvement of the gummy smile using the implant spacer technique. Annals of plastic surgery, 12(1), 1624. https://doi.org/10.1097/00000637-198401000-00004.

[15] Ishida, L. H., Ishida, L. C., Ishida, J., Grynglas, J., Alonso, N., \& Ferreira, M. C. (2010). Myotomy of the levator labii superioris muscle and lip repositioning: a combined approach for the correction of gummy smile. Plastic and reconstructive surgery, 126(3), 1014-1019. https://doi.org/10.1097/PRS.0b013e3181e3b6d4.

[16] Polo M. (2008). Botulinum toxin type A (Botox) for the neuromuscular correction of excessive gingival display on smiling (gummy smile). American journal of orthodontics and dentofacial orthopedics: official publication of the American Association of Orthodontists, its constituent societies, and the American Board of Orthodontics, 133(2), 195-203. https://doi.org/10.1016/j.ajodo.2007.04.033. 